\title{
Uterine smooth muscle tumor of uncertain malignant potential: a case diagnosis, management and follow-up in the department of gynecology- obstetrics II of the Hassan II teaching hospital of Fes in Morocco
}

\section{Alpha Boubacar Conte*, Jihad Jamor, Fatima Zohra Fdili Alaoui, Sofia Jayi, Hikmat Chaara, Moulay Abdelilah Melhouf}

Department of Gynecology - Obstetrics II, Hassan II Teaching Hospital, Sidi Mohamed Ben Abdellah University, Fès, Morocco

Received: 28 January 2020

Revised: 07 March 2020

Accepted: 20 March 2020

*Correspondence:

Dr. Alpha Boubacar Conte,

E-mail: abcconte33@yahoo.com

Copyright: ( ) the author(s), publisher and licensee Medip Academy. This is an open-access article distributed under the terms of the Creative Commons Attribution Non-Commercial License, which permits unrestricted non-commercial use, distribution, and reproduction in any medium, provided the original work is properly cited.

\section{ABSTRACT}

Rarely diagnosed in authors department, uterine smooth muscle tumor of uncertain malignant potential (STUMP) is one of the histologic types of uterine sarcoma. Among women undergoing hysterectomy or myomectomy for a presumed diagnosis of leiomyoma, $0.01 \%$ receive a diagnosis of STUMP. Authors report a case occurring to a patient aged of 55 which was diagnosed, managed successfully and followed-up in authors department.

Keywords: Follow up, Management, Sarcoma, Smooth muscle tumor of uncertain malignant, Uterine tumors

\section{INTRODUCTION}

Rarely diagnosed in authors department, uterine smooth muscle tumor of uncertain malignant potential (STUMP) is one of the histologic types of uterine sarcoma. STUMPs are classified as benign leiomyoma and malignant leiomyosarcoma regarding presence of tumor cell necrosis, cytological atypia and mitotic activity.

STUMP is regarded in World Health Organization (WHO) classification as smooth muscle tumors between benign and malignant criteria. ${ }^{1}$

Among women undergoing hysterectomy or myomectomy for a presumed diagnosis of leiomyoma, $0.01 \%$ receive a diagnosis of STUMP. ${ }^{2}$

The true prevalence of STUMP is difficult to determine due to the rarity of this disease and inconsistency in diagnostic criteria. ${ }^{3}$

\section{CASE REPORT}

A 55-year-old women admitted for uterine abnormal bleeding and chronic pelvic pain evolving for 2 years. Multiparous, in post-menopausal period, with pathological story of high blood pressure. The clinical exam found an abdominopelvic painful, hard and irregular mass arriving midway to the umbilicus with the uterine cervix macroscopically normal and enlarged uterus with a prolapsed lump in the vagina looking to be from to the uterus.

Pelvic ultrasound found an intramural image of $60 \mathrm{~mm}$ with anarchic central vascularization. Pelvic MRI found a uterus augmented of size with $96 \mathrm{~mm}$ intra cavitary mass looking to be a uterine sarcoma. CT-TAP showed a uterus increased of size seat of an endometrial tissue process poorly limited to $75 \mathrm{~mm}$. This process infiltrates the myometrium laterally on the left and comes into contact with some intestine and sigmoid; comes into 
contact with iliacs vessels bilaterally it infiltrates the cervix; the parameters in bilateral and rectum with loss of the border of separation before it infiltrates the bladder. After initial assessment the patient was not operable and the decision was taken to perform an embolization before the surgical procedure. The patient underwent surgery by laparotomy (Total hysterectomy with bilateral adnexectomy). During the procedure, the exploration found: a uterus of increased size no ascites no peritoneal carcinosis, the liver and other intraperitoneal organs were smooth. The surgery was performed without any incident.

The histological examination of the samples taken from the mass delivered by the cervix found a fuso cellular tumor proliferation arranged in intertwined bundles. It has a high cellularity with frank cyto nuclear atypies showing cells with elongated to oval nuclei, irregular contours and dense chromatin, with an eosinophilic cytoplasm. The mitotic count was 9 mitoses/10 CFG. Absence of tumor necrosis area.

The samples taken from the endometrium found large area of necrosis with ghost cells looking like glands which are marked by $\mathrm{CK} 8 / 18$, estrogen and progesterone receptors. Other endometrial samples found area of atypical complex hyperplasia. The appendices, the parameters and the paracervix were free from any invasion. The histological examination described the aspect of a smooth muscle tumor with uncertain malignancy potential (STUMP). Presence of fully necrotic glands (is it a necrotic endometrial polyp or necrotic endometrioid adenocarcinoma after embolization?) to be compared with clinical and para clinical data.

After discussion in a multidisciplinary consultation meeting, an indication of clinical and radiological monitoring was decided. The patient is still under monitoring. She has been clinically monitored twice each 3 months and benefited to a TAP-CT which did not found any recurrence for the first year.

\section{DISCUSSION}

STUMPs represent a group of rare and heterogeneous neoplasms from both a histological and a clinical point of view. ${ }^{4,5}$ The incidence is not well known. ${ }^{6}$ According to Picerno TM and al, 0,01\% of leiomyoma are diagnosed as STUMP. ${ }^{2}$ It also represents $1 / 3$ of uterine sarcomas and $1.3 \%$ of uterine cancers. ${ }^{7}$ There is no clinical specific symptomatology. STUMPs often present with symptoms consistent with a benign leiomyoma.

The symptomatology includes a combination of abnormal uterine bleeding, pelvic mass, or symptoms due to secondary compression and anemia. ${ }^{8}$ Pelvic pain and pelvic pressure sensation or combination of them are also describe. ${ }^{6}$ This case was admitted for uterine abnormal bleeding and pelvic chronic pain and the clinical exam found an abdominopelvic painful, hard and irregular mass arriving midway to the umbilicus.

The age of onset of this disease is similar to that of leiomyoma or leiomyosarcoma, and little is known regarding the specific risk factors that predispose to a diagnosis of STUMP. ${ }^{9}$ Due to the rarity of stumps, there is no demographic data to consolidate the hypothesis based on the age of the occurrence.

But it has been reported by Guntupalli SR et al, that the mean age is 45 and most of patients is in premenopausal period..$^{5}$ In a retrospective study on 6 patients made by Bacanakgil and al, the mean age of the patients was 42 years old and only 1 patient was in post-menopausal period. ${ }^{6}$ In this report the patient was 55 and in postmenopausal period. Which is different from what is reported in some studies. ${ }^{5,6}$ STUMP is often unexpectedly found post-hysterectomy or myomectomy.

It is very difficult to differentiate between benign leiomyoma and malignant sarcomas preoperatively. MRI has been used to differentiate between benign and malignant tumors of the uterus utilizing increased signal intensity; however, little evidence exists to distinguish STUMP from leiomyoma. ${ }^{8}$ Out of study observation authors can also notice that the diagnosis was first suspected by the pelvic ultrasound which showed an intramural image of $60 \mathrm{~mm}$ with anarchic central vascularization. That ultrasound description led us to require a pelvic MRI which description oriented us and consolidated study hypothesis of sarcoma. Even though the differentiation might be difficult between benign and malign tumors of uterus, the imagery has a great place.

In the main idea to show the importance of imagery in the diagnosis of leiomyosarcoma, Bacanakgil et al, compared sonography and MRI findings of 85 leiomyoma and 23 malignant mesenchymal tumor (MMT) and STUMP cases, and reported that presence of single tumor, absence of acoustic shadowing and presence of free fluid are associated with MMT/STUMP. ${ }^{6}$

According to Shapiro A et al, there is no approved standard protocols for the management of patients with suspected STUMP. In the event of STUMP diagnosis in myomectomy specimens, considering the proved possibility of recurrence, hysterectomy represents the gold standard for those women who have completed their childbearing. Successful pregnancies following fertility sparing surgery have been reported however these patients should be adequately informed of the risk of recurrence and a strict follow-up program through clinical and imaging techniques is mandatory. ${ }^{10}$

For the management of this case authors experienced an embolization followed by a total hysterectomy associated to bilateral adnexectomy. It has been suggested that the treatment of choice is the hysterectomy. ${ }^{9}$ If the mass is anarchically vascularized embolization has to be 
requested before any surgical procedure. That embolization will reduce blood vessels flow and make less difficult the surgery.

The diagnosis is found post operatively by the histological analysis of the piece. Uterine smooth muscle tumors have historically been distinct in benign leiomyomas and malignant leiomyosarcomas on the basis of cytological atypia, mitotic rate and presence or absence of tumor cell necrosis (CTCN). ${ }^{11}$ The current criteria for the histopathologic classification of smooth muscle tumors are based on the Stanford Criteria, and they are diagnosed by an assessment for abundant mitosis (10 per 10 HPFs), cellular atypia and presence of areas of coagulative tumor cell necrosis. ${ }^{3,12}$

STUMPs do not fulfill the diagnosis of leiomyosarcoma. Uterine smooth muscle tumors that show some worrisome histologic features (i.e. necrosis, nuclear atypia, or mitoses), but do not meet all diagnostic criteria for leiomyosarcoma, fall into the category of atypical smooth muscle tumors (STUMP). This diagnosis, however, should be used sparingly and every effort should be made to classify a smooth muscle tumor into a specific category when possible. ${ }^{13}$

The prognosis of STUMP is better than that of leiomyosarcomas. Several studies have found a significantly reduced recurrence rate compared to leiomyosarcoma as well as 5-year survival ranging from 92 to $100 \%$ of patients and there is no difference between the rate of patient who underwent hysterectomy and those who underwent myomectomy. 5,14

This present case is still under monitoring and authors opted for clinical monitoring each 3 months for this first year and TAP-CT each 6 months. The current result is good as authors did not find any sign of recurrence and metastasis.

\section{CONCLUSION}

Rare as authors have noticed in the literature, uterine smooth muscle tumor of uncertain malignant potential (STUMP) is a particular entity of uterus tumors. The diagnosis is confirmed on the surgical piece. There is no possibility of preoperative diagnosis and the postoperative should be followed by monitoring whatever be the surgical management.

Authors managed this case by total hysterectomy and bilateral adnexectomy after bilateral hypogastric artery embolization. The monitoring of the patient during the first year showed a good result. More study should be done to assess a prognosis of this entity.

Funding: No funding sources

Conflict of interest: None declared

Ethical approval: Not required

\section{REFERENCES}

1. Tavassoli FA, Devilee P. World Health Organization classification of tumours: tumours of the breast and female genital organs. Lyon: Inter Agency Res Cancer Press; 2003:236-9.

2. Picerno TM, Wasson MN, Gonzalez Rios A and al. Morcellation and the incidence of occult uterine malignancy: a dual-institution review. Int J Gynecol Cancer. 2016;26(1):149-55.

3. Stewart EA, Quade BJ, Laughlin - Tommaso SK. Variants of uterine leimyomas (fibroids), 2017. Available at: http://www.uptodate.com. Accessed on $12^{\text {th }}$ December 2019.

4. Berretta R, Rolla M, Merisio C, Giordano G, Nardelli GB. Uterine smooth muscle tumor of uncertain malignant potential: a three-case report. Int J Gynecol Cancer. 2008;18:1121-6.

5. Guntupalli SR, Ramirez PT, Anderson ML, Milam MR, Bodurka DC, Malpica A. Uterine smooth muscle tumor of uncertain malignant potential: a retrospective analysis. Gynecol Oncol. 2009;113:324-6.

6. Bacanakgil BH, Deveci M, Karabuk E, Soyman Z. Uterine smooth muscle tumor of uncertain malignant potential: clinicopathologic- sonographic characteristics, follow-up and recurrence. World J Oncol. 2017;8(3):76-80.

7. D'all Asta A, Gizzo S, Musaro A, Quaranta M. Uterine smooth muscle tumors of uncertain malignant potential (STUMP): pathology, follow-up and recurrence. Inter $\mathbf{J}$ Clin Experiment Pathol. 2014;7(11):8136-42.

8. Hughes L, Roex A, Parange A. STUMP, a surprise finding in a large fibroid uterus in a 20-year-old woman. Inter J Women's Health. 2018;10:211-4.

9. Schwartz LB, Zawin M, Carcangiu ML, Lange R, McCarthy S. Does pelvic magnetic resonance imaging differentiate among the histologic subtypes of uterine leiomyomata? Fertil Steril. 1998;70(3):580-7.

10. Shapiro A, Ferenczy A, Turcotte R, Bruchim I, Gotlieb WH. Uterine smooth-muscle tumor of uncertain malignant potential metastasizing to the humerus as a high-grade leiomyosarcoma. Gynecol Oncol. 2004;94:818-20.

11. Bell SW, Kempson RL, Hendrickson MR. Problematic uterine smooth muscle neoplasms. A clinicopathologic study of 213 cases. Am J Surg Pathol. 1994;18(6):535-58.

12. Ip PP, Tse KY, Tam KF. Uterine smooth muscle tumors other than the ordinary leiomyomas and leiomyosarcomas: a review of selected variants with emphasis on recent advances and unusual morphology that may cause concern for malignancy. Adv Anat Pathol. 2010;17(2):91-112.

13. Oliva E. Cellular mesenchymal tumors of the uterus: A review emphasizing recent observations. Int $\mathbf{J}$ Gynecol Pathol. 2014;33:374-84. 
14. Duvillard P. Pathologie gynécologique; cas $N^{\circ} 7$. Tumeur musculaire lisse utérine de malignité incertaine (STUMP), Annales de Pathol. 2012;32:211-3.
Cite this article as: Conte $\mathrm{AB}$, Jamor J, Alaoui FZF, Jayi S, Chaara H, Melhouf MA. Uterine smooth muscle tumor of uncertain malignant potential: a case diagnosis, management and follow-up in the department of gynecology-obstetrics II of the Hassan II teaching hospital of Fes in Morocco. Int J Reprod Contracept Obstet Gynecol 2020;9:2598-601. 